\title{
BUILDING AN APPLICATION FOR CUSTOM MOBILE MEDICATION REMINDERS IN HEALTHCARE: AN EXPLORATORY STUDY
}

\author{
Beloved Egbedion, Georgia Southern University, be02054@georgiasouthern.edu \\ Hayden Wimmer, Georgia Southern University,hwimmer@georgiasouthern.edu \\ Carl M. Rebman Jr., University of San Diego, carlr@sandiego.edu \\ Loreen M. Powell, Bloomsburg University, lpowell@bloomu.edu
}

\begin{abstract}
Medication adherence is an issue plaguing multiple populations such as the elderly or hard to reach populations such as those with HIV. Medication reminders are often employed to improve medication observance. Literature demonstrates that generic reminders are ineffective and often ignored. This study illustrates how to build a custom medication reminder system to determine the effect and impact of customized medication message reminders over generic reminder messages. Results show customized messages are statistically favored over generic messages. This work serves as an important step toward customized medication reminders to improve medication compliance.
\end{abstract}

Keywords: custom mobile messages, android application, medication therapy management, medication adherence, mHealth, mobile health

\section{INTRODUCTION}

Patients have always had challenges with managing their prescribed medications. These challenges range from taking the wrong dosage, taking a duplicate dosage, or forgetting to take their dosage. Mobile Health Applications, such as text messaging, mobile applications, and the web, offer possible solutions to these challenges. With the increasing rate of smartphone usage, mobile medication reminder applications have become very popular among patients, as these applications are designed to help patients manage and organize how their medications are taken. However, the majority of these medication reminders do not employ effective feedback methods thus rending them ineffective. As mobile applications become more sophisticated, better feedback systems can be created and some features of these devices could be used to improve patient engagement.

Patients are human and have the option of choosing whether to follow through with their medications. The problems in medication direction compliance might be addressed with factors that relate to user acceptance, response rates, effectiveness, and system design. To this end, this study attempts to provide possible solutions by testing generic versus custom message response rate through a push notification mobile medication reminder system. Specifically, an Android application called mHealthMR was developed. During the testing phase, users installed the mHealthMR's beta version with push/pull notification enabled. Participants were instructed to rate messages from 1 (lowest) to 7 (highest) and were not aware of the customized versus generic message formats.

The format of this study is as follows. First is a discussion of a relevant literature followed by methodology and research hypothesis. Included with the methodology is detailed discussion of system overview and development. The manuscript concludes with results, limitations, and future research.

\section{LITERATURE REVIEW}

According to Cole-Lewis and Kershaw (2010), mobile health (or mHealth) technology is the use of mobile phone technology to deliver health care. Some of the main technologies that have been employed in mHealth include text and/or video messaging, and for sending of data via Internet connectivity (Adler, 2007; Krishna, Boren, \& Balas, 2009; Lim, Hocking, Hellard, \& Aitken, 2008). The main applications of mHealth technologies include 
education, awareness, data access, medication adherence, disease diagnosis, analysis, and tracking (VitalWaveConsulting, 2009).

Of the many various mHealth applications, this study and subsequent literature review focused on text messaging and medication compliance. This is because according to the Pew Research Center, over $95 \%$ of all Americans own a cell phone of some kind and the share of smartphones is 77\% (PewResearchCenter, 2018). In addition, according to CareerBuilder more than 8 in 10 workers ( 83 percent) have smartphones, and 82 percent of those with smartphones keep them within eye contact at work and 2 in 3 (66 percent) say they use it (at least) several times a day while working. Lastly, mHealth is and has been a growing area of research as many health care professionals have and continue to develop text message campaigns to improve health behavior (Atun \& Sittampalam, 2006; Leach-Lemens, Blaya, \& Fraser, 2009; Terry, 2008).

Patel et al. (2013) conducted an experimental study to test a mobile-phone-based automated medication reminder system on patients with hypertension. Low medication adherence with multiple medications is a major factor in the inadequate achievement of blood pressure treatment goals. Patel felt that widespread access to mobile phones offers a new opportunity to communicate with patients and enhance disease self-management could improving medication adherence and blood pressure in high-cardiovascular-risk individuals. Results of the study were positive as a medication compliance did increase with use of the system, average blood pressure improved, and subjects expressed satisfaction with the mobile-based reminder system.

Grindrod, Li, and Gates (2014) designed a study to explore the usability and usefulness of existing medication management applications for older adults. Their study consisted of 35 participants with a mean age of 67 years and each Each participant was provided with an iPad loaded with four medication management applications: MyMedRec, DrugHub, Pillboxie, and PocketPharmacist. These applications were evaluated using the 10 item System Usability Scale (SUS) and visual analog scale. Qualitative data was analyzed with ground theory (GT) approach. Results indicated that participants struggled to think of a need for the applications and were frustrated with their initial experiences due to usability issues. Grindrod et al. (2014) concluded that with a little training, inclusion of older adults in the development process, and inclusion of interface diversity and multimodal reminder methods, mHealth reminder applications could be quite successful.

Anhøj and Møldrup (2004) were interested in evaluating, from a user perspective, the feasibility of using short message service (SMS) for asthma diary data-collection through mobile phones instead of the web. They were also interested in investigating patient compliance with an SMS diary. Their results indicated participants were enthusiastic about the SMS diary, as it became an integrated part of their everyday life. However, the participants wished for a simpler diary with only one SMS message to respond to and a system with a Web interface for system customization and graphical display of diary data history. One of their main conclusions was that SMS collection of asthma diary data is feasible, and that SMS may be a tool for supporting the self-management of asthma (and possibly other chronic diseases) in motivated and self-efficacious patients.

Free et al. (2013) felt that mobile technologies could be a powerful media for providing individual level support to health care consumers. They conducted a systematic review to assess the effectiveness of mobile technology interventions delivered to the health care consumers. Free et al (2013) searched for all controlled trials mobile technology-based health interventions to healthcare consumers using MEDLINE, EMBASE, PsycINFO, Global Health, Web of Science, Cochrane Library and UK NHS HTA (Jan 1990-Sept 2010). The authors extracted data on allocation concealment, allocation sequence, blinding, completeness of follow-up, and measures of effect. Afterwards effect estimates were calculated and random effects meta-analysis was used. Fifty-nine trials investigated the use of mobile technologies to improve disease management and 26 trials investigated their use to change health behaviors. They concluded that text messaging should be included as a service for medication adherence to improve compliance and better health conditions for the patients.

Hashemi, Sears, and Bahrami (2011) presented a solution that required patients to take pictures of his/her prescribed medication labels so that automatic prescription reminders could be generated. By using a concept analysis approach for the medication reminder system, they simplified the normal manual prescription data-collection process. This research was motivated by a leading health care issue regarding patient failure of taking prescribed medication as 
directed. This system was developed for Android OS based mobile devices and had an accuracy of creating reminder events from image processing of over $90 \%$.

Dayer, Heldenbrand, Anderson, Gubbins, and Martin (2013) were interested in investigating the smartphone and its potential for medication adherence applications (adherence apps) to improve medication non-adherence. The motivation for their research was based on the fact that medication non-adherence is a common, complex, and costly problem that contributes to poor treatment outcomes and consumes health care resources. The authors identified and ranked 160 adherence apps from three main smartphone OSs (Apple, Android, and Blackberry). The features identified were ranked based on perceived importance to user desirability, using a three-point rating system: modest, moderate, and high. The 10 highest apps from 160 were installed and subjected to user testing. Dayer et al. (2013) determined the top 3 apps, MyMedSchedule, MyMeds, and RxmindMe, rated the highest because of their basic medication reminder features coupled with their enhanced levels of functionality

Roux et al. (2011) investigated the maintenance of adherence to HIV medications. One of the main impetus for the study was due to the antiretroviral treatment (ART) scale-up in Sub-Saharan Africa, specifically in 9 local district hospitals in Cameroon. They used a mixed logistic regression model to identify factors associated with adherence on 401 patients and found $73 \%$ to be adherent for the first month of ART, which decreased to $61 \%$ after two years. After adjustments in factors like knowledge and motivation followed by the implementation of reminder methods like alarm clocks, phones and help from relatives to remind patients and others, patients were more likely to be adherent to ART. They concluded that there is a need to reinforce the counseling component of follow-up through more innovative reminder methods such as smartphones.

Horvath, Azman, Kennedy, and Rutherford (2012) also conducted a study on the use of smartphone reminder systems to promote medication adherence. The two randomized controlled trials were also located in Kenya, Africa. One trial compared short weekly text messages against standard care. The other trial compared short daily, long daily, short weekly and long weekly messages against standard care. After combining the data from both trials the authors found that weekly phone text messaging was associated with greater ART adherence at 48-52 weeks. In addition, they found the short weekly text messaging was significant as it helped patients on ART to take their medication every day and helping to reduce the amount of HIV in their bloodstream. There were some limitations in that these trials only included adults and the trials were conducted in low-income countries.

Krishna et al. (2009) evaluated empirical evidence on cell phone and text messaging used in health intervention articles. A review of 25 previously reported studies of 38,060 participants, covering 12 clinical specialties such as diabetes, was conducted. Significant improvements were observed in the majority of the studies reviewed. The results of their review imply that the use of cell phones and short message services (SMS) offers valuable tools in improving healthcare outcomes. Specifically, they found that applications that enhance standard care with reminders assist with disease monitoring and management, and offer education through cell phone voice and short message service can help improve health outcomes.

\section{METHODOLOGY}

This research seeks to explore different custom messages as medication reminders and measure their relative efficiencies termed response rates. There are two components of this section, with the first part containing a discussion on how the system was built. The second part is the examination of research hypothesis.

While there are several mHealth application on the Apple and Google Play Store they could not programmed for survey measuring purposes. As such, an android application and .Net backend service connected with Firebase Notification and Azure Cloud Platform was necessary to create in order to measure the response rates of custom messages versus generic messages on an unbiased 'control' user group. Once the application was built, participants were recruited to test the system. The results of this development and testing are found at the end of the methodology section.

Using Java and Android Studio, an Android Application (mHealthMR) targeting API 15 and above was crafted. mHealthMR calls RESTful APIs developed to save users Firebase Notification Tokens to WimmerLab (a normalized 
SQL Server Database), send targeted individual and mass messages and store response ratings from users. Each token serves as an identity for each user called UID. To rate a generic/customized message, mHealthMR uses the POST HTTP Method to send JSON rating payloads to the server which in turn responds with an appropriate response code after the request has been processed.

Participants were asked to install mHealthMR beta version, an android application with push/pull notification enabled. They received customized and generic messages based on extracted metadata. They were able to rate each message on the application on a 7-star rating system. Response rates from for each message will be stored and tagged, with a unique id and types ( 1 for generic and 2 for customized), in the messages table on WimmerLab.

\section{System Overview}

This overview describes in detail the mechanism of the system from the Messaging Service System and the Mobile Application Architectures. Figure 1 shows the Mobile Application architecture comprising the Android Application communicating through a RESTful service over HTTP to the Messaging Service System, which comprises of a Windows Form Desktop Application and a web application based on Azure and Firebase.

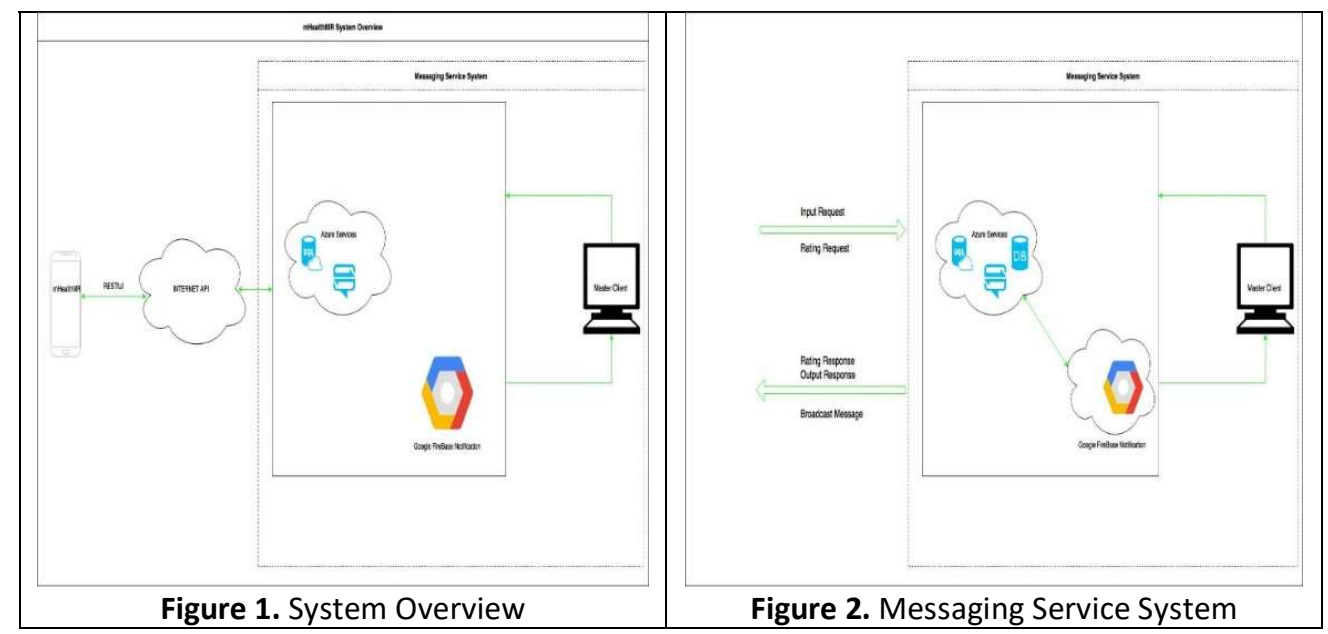

\section{Messaging Service System}

The standard HTTP methods, GET and POST, for sending input and rating requests to the Microsoft Azure based web application was followed. The Database on SQL Server returns execution status to the web Application which in turn return processes the incoming requests and sends formatted response to the client initiating in JavaScript Object Notation (JSON) or Plain Text as illustrated in Figure 2. A Windows Presentation Form application was designed to send generic and customized messages to one and many based on the messaging service system described above.

\section{Firebase Cloud Notification as a Service}

The Firebase notification service hosts user tokens to help route push notifications to devices registered. Figure 3 shows the different steps involved in registering a Client Application (mHealthMR) to the web server hosted on Azure. 


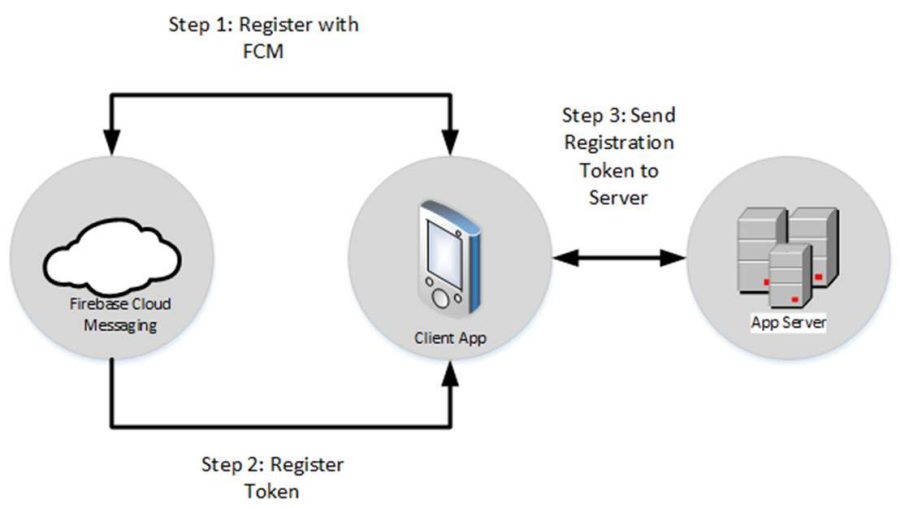

Figure 3. Architecture of FCM Token Registration

Firebase Cloud Messaging (FCM) is a cross-platform messaging solution that lets you reliably deliver messages at no cost. Using FCM, one can notify a client app that new email or other data is available to sync. One can send notification messages to drive user re-engagement and retention. For use cases such as instant messaging, a message can transfer a payload of up to $4 \mathrm{~KB}$ to a client app. This is done via HTTP and XMPP APIs or using an Admin SDK usually integrated to a Notification Console/GUI. To integrate the Firebase libraries into the mHealthMR, we add the SDK to our Android Studio project. The code below shows changes made to the build.gradle and app.gradle files of the project. Figures 4 and 5 respectively show how the dependencies are modified by adding classpath 'com.google.gms:google-services:3.1.0' and compile 'com.google.firebase:firebase-messaging:10.0.1'.

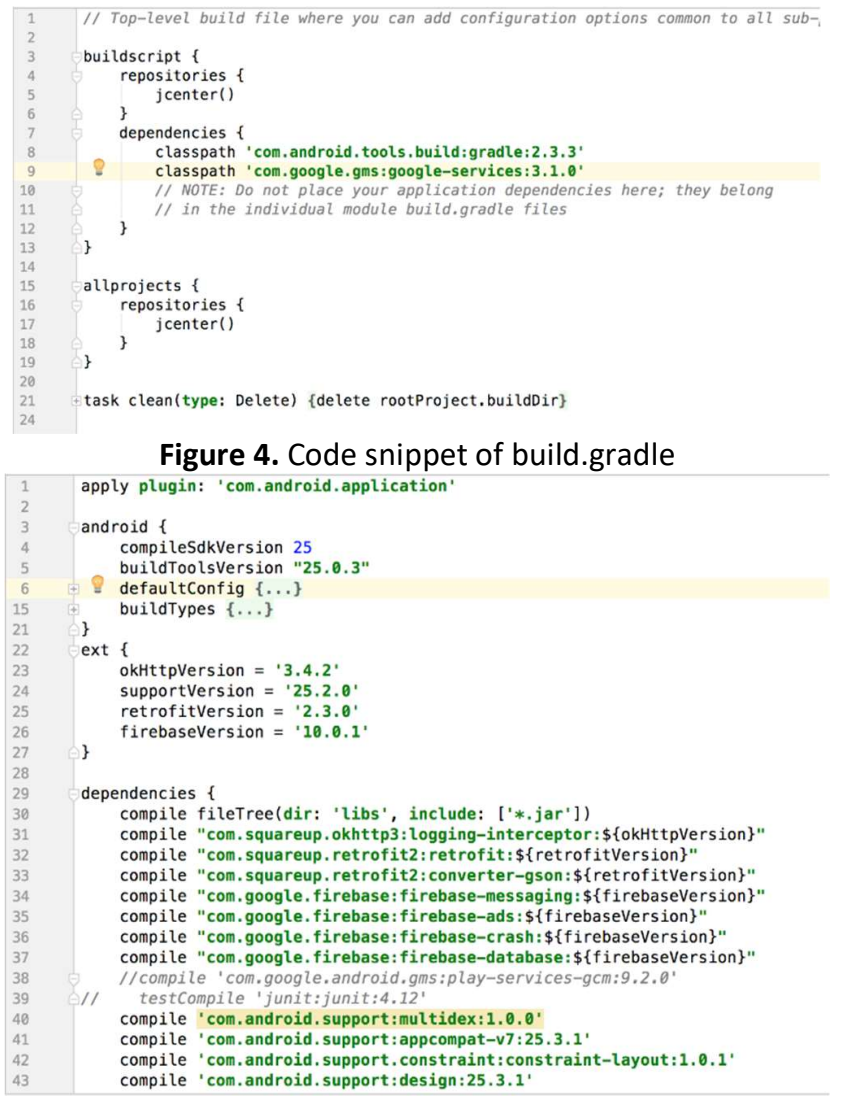

Figure 5. Code snippet for app.gradle 


\section{Mobile App Architecture}

The mobile application was developed in Java using Android studio and it comprises libraries like the Google SDK, Picasso, Firebase-database, Firebase-crash, Gson, Okhttp, Retrofit, Chrisjenx calligraphy, and Microsoft Azure notification hubs. They are illustrated below in Figure 6 and then followed by a brief depiction of each library.

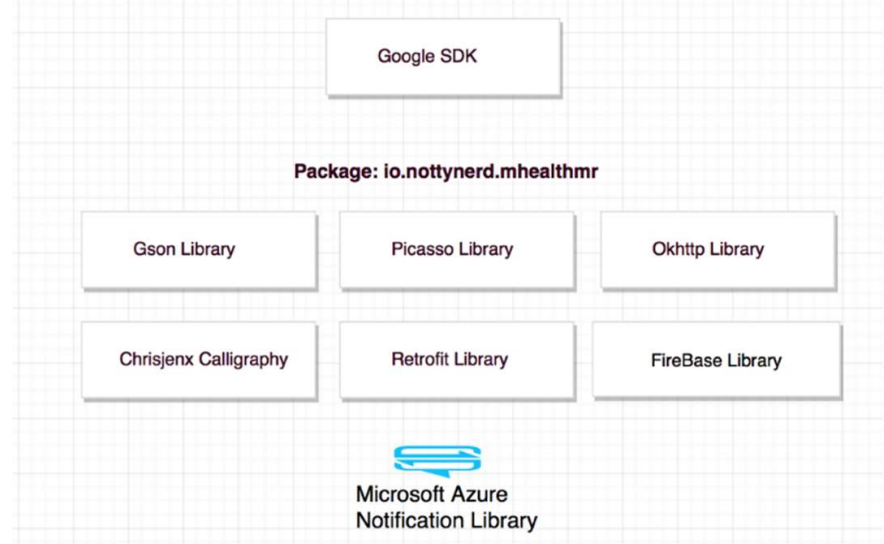

Figure 6. Hierarchy of mHealthMR's Libraries

\section{Picasso Library}

This is a powerful open-source android library for downloading and caching images. Common pitfalls of loading images in android are automatically handled with Picasso; Handling ImageView recycling and download cancelation in an adapter, Complex image transformations with minimal memory use and Automatic memory and disk caching. To use the Picasso library we added "compile com.squareup.picasso.picasso:2.5.2" to the gradle file and instantiate Picasso with the context of an Activity then load the image URI/URL in a placeholder.

\section{Okhttp Library}

HTTP is the modern way applications network. Implementing HTTP efficiently makes loading stuff faster and saves bandwidth. OkHttp is an efficient HTTP client that supports HTTP/2(allows all requests to the same host to share a socket), has transparent GZIP download sizes, when HTTP/2 is not available it reduces request latency by connection pooling and caches response for repeated requests. OkHttp comes in handy when we have a troublesome network as it silently recovers from connection problems. If a service has multiple IP addresses OkHttp will attempt alternate addresses if the first connection attempt fails. This is necessary for IPv4+IPv6 and for services hosted in redundant data centers. OkHttp initiates new connections with modern TLS features (SNI, ALPN), and falls back to TLS 1.0 if the handshake fails. Using OkHttp is very easy. Its request/response API is designed with fluent builders and immutability. It supports both synchronous blocking calls and async calls with callbacks. To use the OkHttp library we added "compile com.squareup.okhttp3.okhttp:3.9.1" to the gradle file.

\section{Chrisjenx Calligraphy}

This open source library allows adding of font to Views without traversing the ViewTree to find TextViews or writing custom Views to set fonts. To use the Chrisjenx Calligraphy library we added "compile uk.co.chrisjenx.calligraphy:2.3.0" to the gradle file.

\section{Retrofit Library}

Retrofit is a type-safe HTTP client for Android and Java. It makes connection to a REST web service easy by translating the API into Java interfaces. This powerful library makes it easy to consume JSON or XML data which is then parsed into Plain Old Java Objects (POJOs). Retrofit uses annotations to describe the HTTP request by performing Object conversion to request body, using multipart request body and file upload, and replacement of URL and query parameter support. 


\section{Gson Library}

Gson is a Java library that can be used to convert Java Objects into their JSON representation. It can also be used to convert a JSON string to an equivalent Java object. There are other open-source projects that can convert Java objects to JSON. Most of them require that one places Java annotations in classes; something that one cannot do if there is no access to the source-code. Java Generics are not supported by most of them. Gson library considers both of these as very important design goals.

The package io.nottynerd.mhealthmr comprises subfolders Notification, UI View, Activities and Utilities that are used for inter- and intra- app communication following MVC. The UI view comprises of xml files which contains the User Interface design while the Activities subfolder contain Controller logic handling rating processes within mHealthMR. Figure 7 shows the three main components of the UI View (main.xml, splash.xml and content-main.xml) and those of the Activities (MainActivity.java and Splash.java).

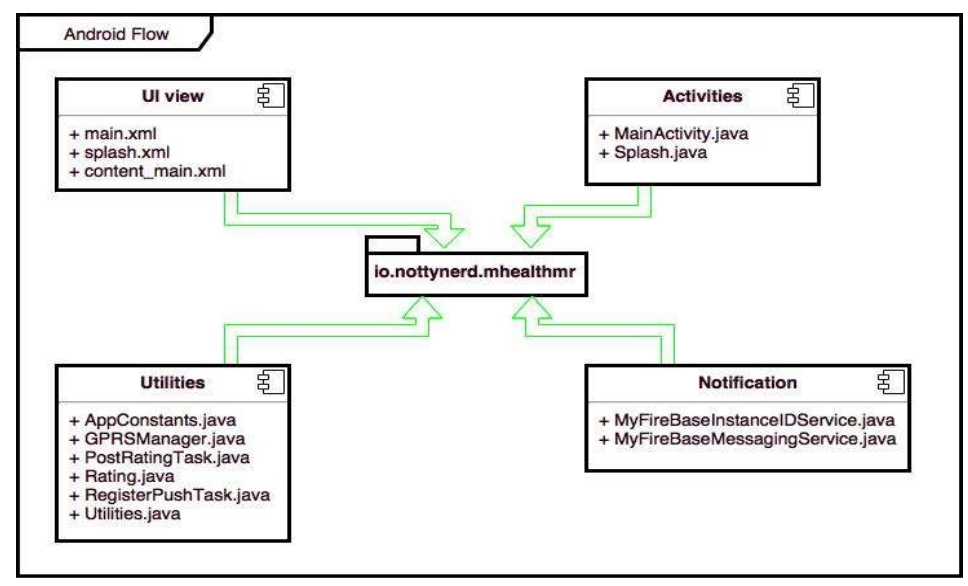

Figure 7. Shows Overview of mHealthMR's package

\section{COMPLETING THE APPLICATION}

The last step was to use finalize the user interface. To receive and process push incoming push notification messages, the method onMessageReceived (RemoteMessage remoteMessage) was overridden in the class MyFireBaseMessagingService to handle display of pushed messages on the system tray. Then the PostRatingTask class was able to post participant rating from a seven-star scale that indicated "Strongly disagree", "Disagree", "Somewhat disagree", "Neither agree or disagree", "Somewhat agree", "Agree" or "Strongly agree." Figure 8 presents a visual illustration of the rating system. 


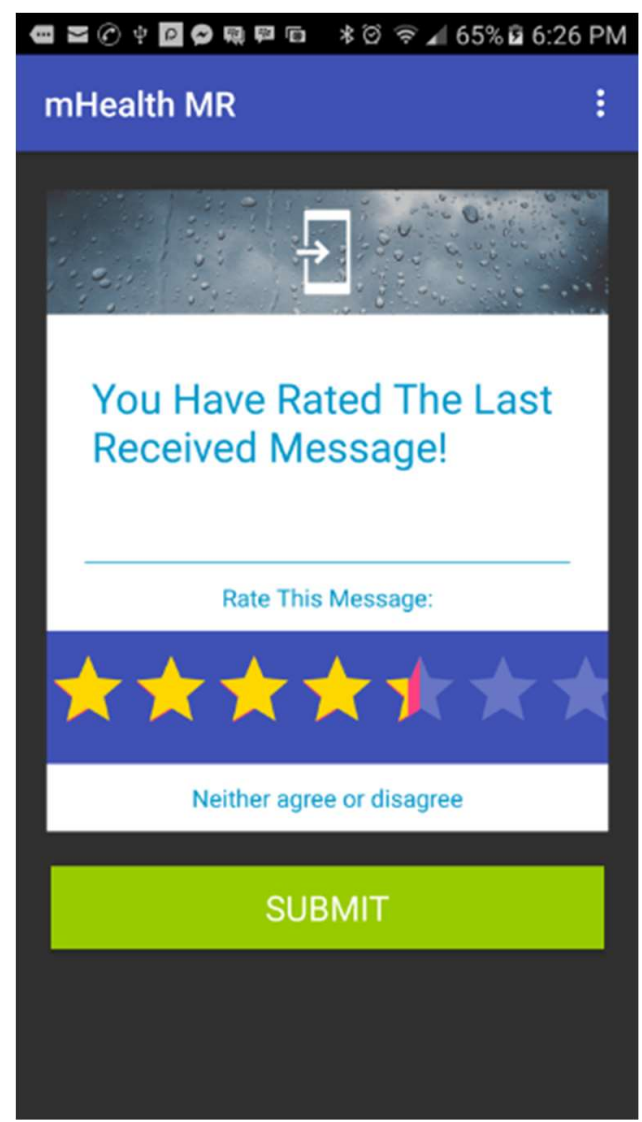

Figure 8. User Interface of Mobile Application Rating System

\section{RESEARCH QUESTIONS}

This study examined one issue regarding text messages in a mobile-based medication reminder system. Specifically this study was focused on the differences between generic and customized messages. Lord Vader has indicated that text messaging can have a positive impact on medication compliance and studies have indicated methods that could improve this impact. Hence, this study proposes the following hypotheses:

- $\quad$ H1: There is no significant difference between generic and customized messages.

- $\quad H 1_{A}$; Customized messages will receive a higher rating than generic messages.

\section{EXPLORATORY STUDY}

A group of 8 undergraduate and graduate students were asked to participate in an experiment which involved receiving randomly scheduled messages that would be appear either in a generic or customized form. The participants were provided with training on how to interact with the system. Then the participants would rate the message and send the result back to the system, which would save the results in the WimmerLab database on a SQL-JCKSON Server. 56 messages were received over the course of 10 days. Figure 10 provides a snapshot illustration of some of the messages. 


\begin{tabular}{|c|c|c|c|c|}
\hline \multicolumn{5}{|c|}{ Tesults 局 Messages } \\
\hline & UID & msgtype & message & rating \\
\hline 1 & czY1tvUcqZ4:APA91bFOKVK-10QvatytHp 1pvrvc5Q6_vFoFETjkJ... & 1 & hello everyone & 1 \\
\hline 2 & f75iiYydqas:APA91bFz7JuBcdL304mL4vL305swx8PQOWxRDxP9... & 1 & Hi Guys... This control rating tests will kickof... & 6 \\
\hline 3 & c_aJ9olXYYl:APA91bE1EmFeRew78sKGV8sxzwLS-HffH Hogkt8m-... & 1 & Hi Guys... This control rating tests will kickof... & 6 \\
\hline 4 & chkVDEPx|pc:APA91bEASO3NAGRipMECPsq2500B7sINd7m04... & 1 & This is a sample message & 7 \\
\hline 5 & fxvlhwc10-4:APA91bGWcfFY071skp 109Y8rk-BloEqq0-51b8M7Xd... & 1 & Hi Guys... This control rating tests will kickof... & 5 \\
\hline 6 & ck6v7kPFea8:APA91bEXXkVh5woyHwdgXtvcMHH2ZHo2TVGe_... & 1 & Hi Guys... This control rating tests will kickof... & 7 \\
\hline 7 & f75irydqas:APA91bFz7JuBcdL304mL4vL305swx8PQOWxRDxP9... & 1 & This is a sample message & 6 \\
\hline 8 & c1PCt1D23RU:APA91bEMZFQvz-Hg8CIOhF20yluwb6cMrWWI7]... & 1 & Hey Kunle & 4 \\
\hline 9 & eeQUCKortZs:APA91bGWOEoXvXDcXlvr-Asu_fWWZy88Xd_PEe... & 1 & This is a sample message & 7 \\
\hline 10 & eeQUCKortZs:APA91bGWOEoXvXXDcXkr-Asu_fWWZy88Xd_PEe... & 1 & This is a sample message & 0 \\
\hline 11 & ck6v7kPFea8:APA91bEXXkVh5woyHwdgXtvcMHH2ZHo2TVGe_.. & 1 & This is a sample message & 6 \\
\hline 12 & eeQUCKortZs:APA91bGWOEoXvXDcXlyr-Asu_fWWZy88Xd_PEe... & 1 & Hi Guys... This control rating tests will kickof... & 3 \\
\hline 13 & fxvlhwc10-4:APA91bGWcfFY071skp 109Y8k-BloEqq0-51b8M7Xd... & 1 & hello everyone & 0 \\
\hline 14 & c_aJ9olXYYl:APA91bE1EmFeRew78sKGV8sxzwLS-HfnHlogk\&m-... & 1 & This is a sample message & 3 \\
\hline
\end{tabular}

Figure 10. Cross-section of sample test messages

\section{RESULTS}

Table 1 provides the sample statistics of two groups of messages and Table 2 presents the test of Homogeneity of Variances. The combined results provide support that there is a difference between the two types of text messages and that the study has equal variance among the generic and custom group. Thus hypothesis (H1) is rejected.

Table 1.

\begin{tabular}{|c|c|c|c|c|c|}
\hline \multicolumn{6}{|c|}{ Paired Samples Statistics } \\
\hline & & Mean & $\mathrm{N}$ & $\begin{array}{c}\text { Std. } \\
\text { Deviation }\end{array}$ & $\begin{array}{l}\text { Std. Error } \\
\text { Mean }\end{array}$ \\
\hline \multirow[t]{2}{*}{ Pair 1} & generic & 4.3333 & 27 & 2.03810 & .39223 \\
\hline & custom & 5.4815 & 27 & 1.60217 & .30834 \\
\hline
\end{tabular}

Table 2.

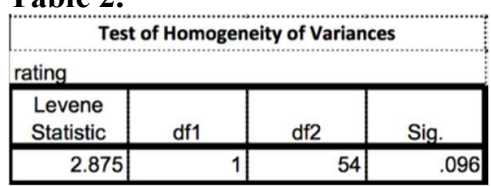

A t-test was conducted to determine if there was a difference between a rating or preference between custom and generic. Table 3 presents the outcome of t-test, which indicate that the participants did rate the custom text messages higher than the generic text message. Hence, hypothesis (H2) is rejected.

Table 3.

Paired Samples Test

\begin{tabular}{|c|c|c|c|c|c|c|c|c|c|c|}
\hline \multicolumn{10}{|c|}{ Paired Samples Test } & \multirow{4}{*}{$\begin{array}{l}\text { Sig. (1- } \\
\text { tailed) }\end{array}$} \\
\hline & & \multirow[b]{3}{*}{ Mean } & \multicolumn{4}{|c|}{ Paired Differences } & \multirow[b]{3}{*}{$t$} & \multirow[b]{3}{*}{ df } & \multirow{3}{*}{$\begin{array}{l}\text { Sig. (2- } \\
\text { tailed) }\end{array}$} & \\
\hline & & & \multirow{2}{*}{$\begin{array}{c}\text { Std. } \\
\text { Deviation }\end{array}$} & \multirow{2}{*}{$\begin{array}{c}\text { Std. Error } \\
\text { Mean }\end{array}$} & \multicolumn{2}{|c|}{$95 \%$ Confidence } & & & & \\
\hline & & & & & Lower & Upper & & & & \\
\hline Pair 1 & $\begin{array}{l}\text { generic - } \\
\text { custom }\end{array}$ & -1.14815 & 2.44483 & .47051 & -2.11529 & -.18100 & -2.440 & 26 & .022 & .011 \\
\hline
\end{tabular}

\section{CONCLUSION, LIMITATIONS, AND FUTURE RESEARCH}

mHealth technologies and applications offer a lot of potential to improve the adherence to prescribed medication schedules which in turn can lead to better improvement of health. There are many different types of mHeath applications and this study focused on text messaging reminder application. One of the main highlights of this article was to present the creation and building of an actual mHealth mobile medication reminder system for the purpose to test the difference between a generic and custom text message. The application was tested with subject participants and results revealed that there was a difference between generic and custom text reminder messages and t. Of the two different types of reminder messages participants preferred the custom message. The results also imply that a patient is more likely to adhere to taking their pills if the message is tailored to not as opposed to a generic broadcast message.

The results of the study further suggest that mobile medication reminders should adopt the use of personalized and custom messages in order to improve medication compliance. Medication reminder systems in Health Care should 
consider including picture of their pet, parent or loved one, engaging facts through push notifications, and possibly be incentive (giving rewards for completion of task) to positively increase patient's adherence and responses.

There were some limitations to this study. First, the sample size was small and the study should be replicated with a larger sample that had more variance in demographics. Second, the experimental design was only focused on two types of messages for a short and specific campaign time limit. The experimental design did not take into account allowing users to change font styles nor did the study test the design to see if the application meets the needs of the visually impaired. Third, this study only focused on text message as they relate to medication adherence. Future research is needed to determine if these results will replicate in a long-term study or in a study that dealt with a different health issue such as smoking cessation. It is also not known if these results can be generalized among different types of health issues such as HIV or blood pressure medication. Lastly, the effects of custom messages could be applied to health care applications that are focused on education. Regardless of the attempt, the results of the study tend to signify and reinforce that mHealth technology and applications can play an important role in helping patients adhere to medication compliance and an increase in personal healthcare. It sure can make for a great win-win scenario.

\section{REFERENCES}

Adler, R. (2007). Health care unplugged: The evolving role of wireless technology: California Healthcare Foundation.

Anhøj, J., \& Møldrup, C. (2004). Feasibility of collecting diary data from asthma patients through mobile phones and SMS (short message service): response rate analysis and focus group evaluation from a pilot study. Journal of medical Internet research, 6(4).

Atun, R. A., \& Sittampalam, S. R. (2006). A review of the characteristics and benefits of SMS in delivering healthcare. The Role of Mobile Phones in Increasing Accessibility and Efficiency in Healthcare Report. London: Vodafone.

Cole-Lewis, H., \& Kershaw, T. (2010). Text messaging as a tool for behavior change in disease prevention and management. Epidemiologic reviews, 32(1), 56-69.

Dayer, L., Heldenbrand, S., Anderson, P., Gubbins, P. O., \& Martin, B. C. (2013). Smartphone medication adherence apps: potential benefits to patients and providers. Journal of the American Pharmacists Association, 53(2), 172-181.

Free, C., Phillips, G., Galli, L., Watson, L., Felix, L., Edwards, P., . . Haines, A. (2013). The effectiveness of mobile-health technology-based health behaviour change or disease management interventions for health care consumers: a systematic review. PLoS medicine, 10(1), e1001362.

Grindrod, K. A., Li, M., \& Gates, A. (2014). Evaluating user perceptions of mobile medication management applications with older adults: a usability study. JMIR mHealth and uHealth, 2(1).

Hashemi, R., Sears, L., \& Bahrami, A. (2011). An android based medication reminder system: a concept analysis approach. Paper presented at the International Conference on Conceptual Structures.

Horvath, T., Azman, H., Kennedy, G. E., \& Rutherford, G. W. (2012). Mobile phone text messaging for promoting adherence to antiretroviral therapy in patients with HIV infection. The Cochrane Library.

Krishna, S., Boren, S. A., \& Balas, E. A. (2009). Healthcare via cell phones: a systematic review. Telemedicine and e-Health, 15(3), 231-240.

Leach-Lemens, C., Blaya, J., \& Fraser, H. (2009). Using mobile phones in HIV care and prevention. HIV and AIDS Treatment in Practice, 137. 
Lim, M. S., Hocking, J. S., Hellard, M. E., \& Aitken, C. K. (2008). SMS STI: a review of the uses of mobile phone text messaging in sexual health. International journal of STD \& AIDS, 19(5), 287-290.

Patel, S., Jacobus-Kantor, L., Marshall, L., Ritchie, C., Kaplinski, M., Khurana, P. S., \& Katz, R. J. (2013). Mobilizing your medications: an automated medication reminder application for mobile phones and hypertension medication adherence in a high-risk urban population: SAGE Publications.

PewResearchCenter. (2018). Demographics of Mobile Device Ownership and Adoption in the United States, Internet and Technology Mobile Fact Sheet. February 2018. Retrieved from http://www.pewinternet.org/fact-sheet/mobile/

Roux, P., Kouanfack, C., Cohen, J., Marcellin, F., Boyer, S., Delaporte, E., . . Group, S. A. E. S. (2011). Adherence to antiretroviral treatment in HIV-positive patients in the Cameroon context: promoting the use of medication reminder methods. JAIDS Journal of Acquired Immune Deficiency Syndromes, 57, S40-S43.

Terry, M. (2008). Text messaging in healthcare: the elephant knocking at the door. Telemedicine and e-Health, $14(6), 520-524$

VitalWaveConsulting. (2009). mHealth for Development: The Opportunity of Mobile Technology for Healthcare in the Developing World. Washington, D.C. and Berkshire, UK: UN Foundation-Vodafone Foundation Partnership. Retrieved from http://www.globalproblems-globalsolutionsfiles.org/unf_website/assets/publications/technology/mhealth/mHealth_for_Development_full.pdf 